\title{
First-principles prediction of high Curie temperature for ferromagnetic bcc-Co and bcc- FeCo alloys and its relevance to tunneling magnetoresistance
}

M. Ležaić, Ph. Mavropoulos, and S. Blügel

Citation: Appl. Phys. Lett. 90, 082504 (2007); doi: 10.1063/1.2710181

View online: https://doi.org/10.1063/1.2710181

View Table of Contents: http://aip.scitation.org/toc/apl/90/8

Published by the American Institute of Physics

\section{Articles you may be interested in}

Electronic structure, exchange interactions, and Curie temperature of $\mathrm{FeCo}$

Journal of Applied Physics 85, 4833 (1999); 10.1063/1.370036

Tuning the Curie temperature of FeCo compounds by tetragonal distortion

Applied Physics Letters 103, 102404 (2013); 10.1063/1.4819773

Commentary: The Materials Project: A materials genome approach to accelerating materials innovation APL Materials 1, 011002 (2013); 10.1063/1.4812323

First-principles exchange interactions in $\mathrm{Fe}, \mathrm{Ni}$, and $\mathrm{Co}$

Journal of Applied Physics 85, 4827 (1999); 10.1063/1.370495

Giant tunneling magnetoresistance up to $410 \%$ at room temperature in fully epitaxial $\mathrm{Co} / \mathrm{MgO} / \mathrm{Co}$ magnetic tunnel junctions with bcc Co(001) electrodes

Applied Physics Letters 89, 042505 (2006); 10.1063/1.2236268

A review of high magnetic moment thin films for microscale and nanotechnology applications

Applied Physics Reviews 3, 011301 (2016); 10.1063/1.4941311

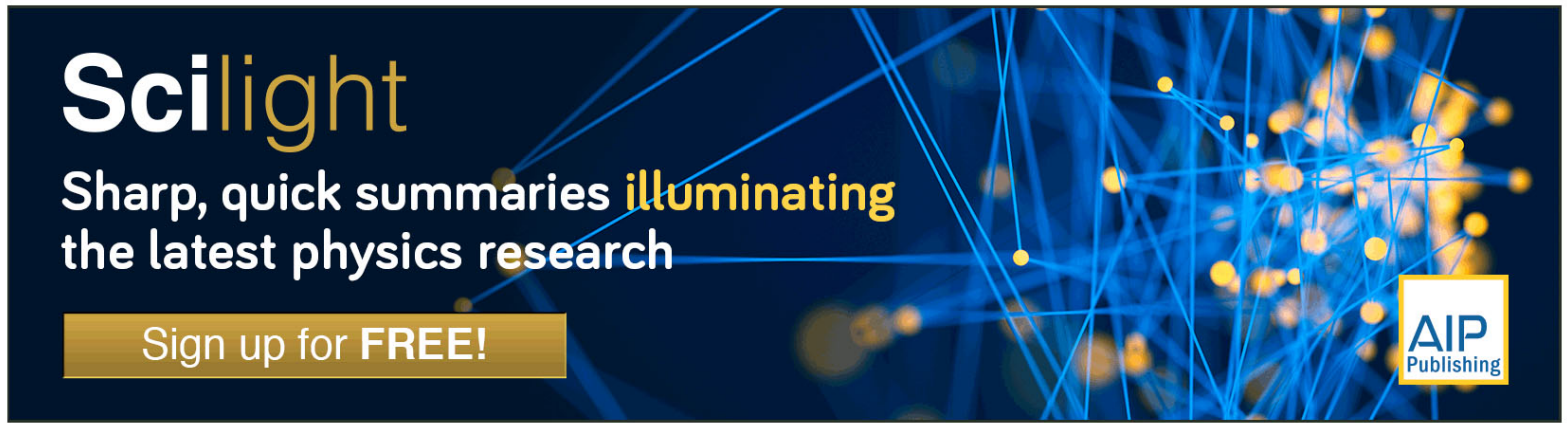




\title{
First-principles prediction of high Curie temperature for ferromagnetic bcc-Co and bcc-FeCo alloys and its relevance to tunneling magnetoresistance
}

\author{
M. Ležaić, ${ }^{\text {a) }} \mathrm{Ph}$. Mavropoulos, ${ }^{\text {b) }}$ and S. Blügel \\ Institut für Festkörperforschung, Forschungszentrum Jülich, D-52425 Jülich, Germany
}

(Received 18 December 2006; accepted 25 January 2007; published online 21 February 2007)

\begin{abstract}
The authors determine from first principles the Curie temperature $T_{C}$ for bulk Co in the hcp, fcc, bcc, and body-centered-tetragonal (bct) phases, for FeCo alloys, and for bcc and bct Fe. For bcc Co, $T_{C}=1420 \mathrm{~K}$ is predicted. This would be the highest Curie temperature among the Co phases, suggesting that bcc- $\mathrm{Co} / \mathrm{MgO} / \mathrm{bcc}-\mathrm{Co}$ tunnel junctions offer high magnetoresistance ratios even at room temperature. The Curie temperatures are calculated by mapping $a b$ initio results to a Heisenberg model, which is solved by a Monte Carlo method. (C) 2007 American Institute of Physics. [DOI: 10.1063/1.2710181]
\end{abstract}

In the past few years we are witnessing a compelling race of different research groups ${ }^{1-4}$ hunting the maximum tunneling magnetoresistance ratio (TMR) of magnetic tunnel junctions $^{5}$ (MTJs) at room temperature (RT). MTJs, made of two ferromagnetic electrodes separated by an insulating barrier, open vistas to a wide field of technological applications, in particular, in nonvolatile magnetic random access memory or recording heads for ultrahigh-density hard-disk drives.

After the initially reported observations of TMR at room temperature ${ }^{6}$ in $\mathrm{Al}_{2} \mathrm{O}_{3}$-based junctions, reaching $30 \%$ at $4.2 \mathrm{~K}$ and $18 \%$ at $300 \mathrm{~K}$, giant values of over $150 \%$ at RT have been experimentally achieved for fully epitaxial $\mathrm{MgO}$ based MTJs. ${ }^{1-4,7}$ Theory predicts (at temperature $T=0$ ) extremely high TMR of over $1000 \%$ for $\mathrm{Fe} / \mathrm{MgO} / \mathrm{Fe}$ (Ref. 8) and even higher for epitaxial bcc-Co/MgO/bcc-Co junctions. ${ }^{9}$ bcc Co is a metastable phase and cannot be grown as a single crystal. Recently, however, it has been possible to grow bec $\mathrm{Co}$ in contact with $\mathrm{MgO},{ }^{7,10,11}$ and epitaxial bcc-Co/MgO/bcc-Co(100) MTJs have shown a high TMR, unusually stable ${ }^{11}$ with $T$.

In most MTJs there is a large difference of the TMR between cryogenic and room temperatures. The TMR (relative change of resistance when the magnetic leads are coupled either ferromagnetically or antiferromagnetically) depends on the details of the electronic structure, such as the spin polarization of the ferromagnet at the Fermi level $E_{F}$ (Ref. 12) and the spin-dependent symmetry of the states at $E_{F}{ }^{7,13}$ At $T>0$, magnetic excitations mix the two spin channels, decreasing the TMR. ${ }^{14}$ One can infer that a high $T_{C}$ and spin stiffness entail a temperature stability of the magnetic structure and of the TMR. In this letter we investigate the Curie temperature of bcc-Co and $\mathrm{Fe}_{1-x} \mathrm{Co}_{x}$ alloys and relate it to fcc $\mathrm{Co}$, hep $\mathrm{Co}$, and bcc Fe, concluding that a high (calculated) (Ref. 20) $T_{C}$ of bcc Co is responsible for the temperature stability ${ }^{11}$ of $\mathrm{TMR}$ in $\mathrm{Fe} / \mathrm{bcc}-\mathrm{Co} / \mathrm{MgO} /$ bcc-Co/Fe junctions.

We calculate the Curie temperature using a standard recipe: the adiabatic approximation for the calculation of magnon spectra. ${ }^{15,16} A b$ initio total-energy results are mapped to the classical Heisenberg model,

\footnotetext{
${ }^{a}$ Electronic mail: m.lezaic@fz-juelich.de

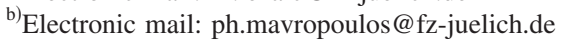

$$
H=-\sum_{i, j ; i \neq j} J_{i j} \mathbf{e}_{i} \cdot \mathbf{e}_{j}
$$

Here, $J_{i j}$ are the exchange constants between the magnetic moments at sites $i$ and $j$, and $\mathbf{e}_{i}$ is a unit vector along the moment of atom $i . T_{C}$ was calculated within this model by a Monte Carlo method (using 1728 atoms in the supercell) by locating the susceptibility peak.

The $a b$ initio results are calculated within the generalized gradient approximation ${ }^{17}$ to density-functional theory. We employ the full-potential Korringa-Kohn-Rostoker (KKR) Green function method, ${ }^{18}$ using the coherent potential approximation for the electronic structure of the $\mathrm{Fe}_{1-x} \mathrm{Co}_{x}$ random alloys. The exchange constants $J_{i j}$ are related to the Green function ${ }^{16}$ assuming an infinitesimal direction change of the moments at sites $i$ and $j$. For $\mathrm{Fe}$ and Co we also compare to a complementary method, where finite-angle static magnon dispersion relations, $J(\mathbf{q})$, are calculated on a dense mesh of $\mathbf{q}$ points in the Brillouin zone within the fullpotential linearized augmented plane-wave method (FLAPW). ${ }^{19}$ From $J(\mathbf{q})$, the real-space constants $J_{i j}$ are found via a Fourier transform. ${ }^{15}$ The two methods give the same trends. We stress that the trends are important for our conclusions and not the absolute values of $T_{C}$, which are off the experimental value (bcc Fe: $T_{C}=1043 \mathrm{~K}$; fcc Co: $T_{C}$ $=1403 \mathrm{~K})^{20,21}$ by $\sim 10 \%$.

Our calculations include bcc Fe and Co, body-centeredtetragonal (bct) $\mathrm{Fe}$ and $\mathrm{Co}$ [considering growth on $\mathrm{MgO}(001)]$, the disordered alloys $\mathrm{Fe}_{0.75} \mathrm{Co}_{0.25}, \mathrm{Fe}_{0.50} \mathrm{Co}_{0.50}$, and $\mathrm{Fe}_{0.75} \mathrm{Co}_{0.25}$, and the ordered alloy $\mathrm{FeCo}$ (in the $\mathrm{CsCl}$ structure). In order to see the effect of compression on the magnetic properties, we calculated the results at two lattice parameters: $a=2.30$ a.u. $=2.804 \AA$, corresponding to the calculated equilibrium lattice parameter of bcc $\mathrm{Co}$, and $a$ $=2.40$ a.u. $=2.857 \AA$. We find for hcp Co an equilibrium lattice parameter $a=2.488 \AA$, with $c / a=1.623$ (experimental values are $a=2.51 \AA, c / a=1.623$ ), for fcc Co $a=3.52 \AA$ (experimental value is $a=3.54 \AA$ ), for bcc Co $a=2.804 \AA$ (extrapolated experimental value is $a=2.819 \AA),{ }^{22}$ and for bcc Fe $a=2.825 \AA$ (experimental value is $a=2.867 \AA$ ). For Co and $\mathrm{Fe}$ on $\mathrm{MgO}(001)$ we find a mismatch of $5 \%$ in the surface lattice parameter (compared to $a_{\mathrm{MgO}} / \sqrt{2}=2.978 \AA$ ), resulting in a ratio $c / a=0.857$ for bet $\mathrm{Co}$ and $c / a=0.909$ for 


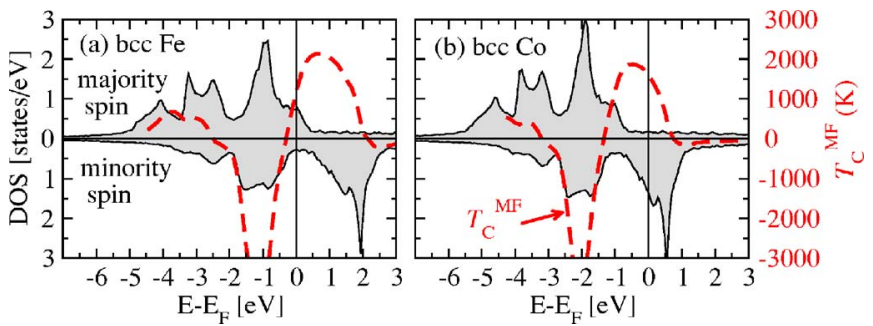

FIG. 1. (Color online) Density of states (solid line) and mean-field ordering temperature (dashed line) for (a) bcc Fe and (b) bcc Co.

bct Fe. The ground state spin moments per Co atom, $M^{\text {hcp }}$ $=1.6, M^{\mathrm{fcc}}=1.65$, and $M^{\mathrm{bcc}}=1.75 \mu_{B}$, change with the crystal structure by less than $10 \%$. The density of states (DOS) of bcc Co, shown in Fig. 1, exhibits the typical bimodal behavior of the bcc lattice with bonding and antibonding states. The spin polarization at $E_{F}, P=-82 \%$, is negative and has a larger (absolute) value than for bcc Fe $(P=52 \%)$. Our calculated band structure agrees with the one of Ref. 23 .

Our results on the magnetic properties are summarized in Table I. A striking effect is that the first-neighbor exchange constants and the $T_{C}$ increase with the Co concentration in $\mathrm{Fe}_{1-x} \mathrm{Co}_{x}$ alloys, with a maximum of about $T_{C}=1670 \mathrm{~K}$ for the ordered FeCo alloy and a $T_{C} \approx 1400 \mathrm{~K}$ for bec and bet Co. In contrast, bct Fe suffers a $20 \%$ decrease of $T_{C}$ compared to bcc Fe. We also find interesting trends in the magnetic moments and in the lattice-parameter dependence of the exchange constants. As the Co concentration increases, the local moment of $\mathrm{Fe}$ becomes larger, climbing from $M_{\mathrm{Fe}}$ $\approx 2.2 \mu_{B}$ in pure $\mathrm{Fe}$ to about $2.6 \mu_{B}$ in $\mathrm{Fe}_{0.25} \mathrm{Co}_{0.75}$. The Co moment is comparatively independent of concentration, about $1.73-1.80 \mu_{B}$. Moreover, the Fe-Fe first-neighbor exchange constants $J_{1}(\mathrm{Fe}-\mathrm{Fe})$ are strongly dependent on the lattice parameter for low Co concentrations $x$ but much less so for high $x$; the $\mathrm{Co}-\mathrm{Co}$ and $\mathrm{Fe}-\mathrm{Co}$ exchange constants,
$J_{1}(\mathrm{Co}-\mathrm{Co})$ and $J_{1}(\mathrm{Fe}-\mathrm{Co})$, are much less affected by the lattice parameter at any $x$. The same behavior is found for the longer-distance exchange constants (not shown here). This leads to a strong dependence of $T_{C}$ on the lattice parameter for Fe-rich alloys and a weak dependence for Co-rich alloys.

The change of the Fe properties upon increasing the Co concentration can be explained by observing that Fe progressively changes character from a weak ferromagnet (i.e., both majority- and minority-spin $d$ states, $d^{\uparrow}$ and $d^{\downarrow}$, are only partly occupied for pure $\mathrm{Fe})$ to a strong ferromagnet $\left(d^{\uparrow}\right.$ states become fully occupied); Co itself is a strong ferromagnet. The progressive change occurs because the $d(\mathrm{Fe})-d(\mathrm{Co})$ hybridization is weaker than the $d(\mathrm{Fe})-d(\mathrm{Fe})$ hybridization, as the Co $d$ states are more localized (they are deeper in the atomic potential well) than the Fe $d$ states. As a result, an increased coordination with Co results in an increase of the Fe moment and exchange splitting, driving the Fe $d^{\uparrow}$ states lower and the Fe $d^{\downarrow}$ states higher in energy. The Co moment, on the other hand, is rather stable (around $1.8 \mu_{B}$ ), since the Co $d^{\uparrow}$ states are always fully occupied. ${ }^{24}$ The average moment per unit cell, $\bar{M}$, first rises upon alloying with $\mathrm{Co}$, due to the rapid increase of the $\mathrm{Fe}$ local moment, then peaks and drops for higher Co content (because of the comparatively lower Co moment). This peak of $\bar{M}$ in Fe-Co alloys is long known, ${ }^{25-28}$ and is related to the triangular shape of the Slater-Pauling curve at the center of the $3 d$ series; ${ }^{27}$ densityfunctional calculations reproduce this behavior, although they underestimate the local Fe moment by about $10 \%$ in these alloys. ${ }^{28}$

In a weak ferromagnet the magnetic properties can be easily affected by structural perturbations (compression or tetragonalization) because an extended part of the Fermi surface has $d^{\uparrow}$ and $d^{\downarrow}$ characters, allowing for $d^{\uparrow}-d^{\downarrow}$ charge transfer upon the structural perturbation. Such $d^{\uparrow}-d^{\downarrow}$ transfer can affect the local moment, but even more the spin suscep-

TABLE I. Calculated (within KKR) magnetic moments (in $\mu_{B}$ ), first-neighbor exchange constants $J_{1}$ (in meV), and $T_{C}$ (in K) of bcc FeCo alloys, bcc Fe and $\mathrm{Co}$, bct Fe and $\mathrm{Co}$, and fcc and hcp Co. The bcc structures are considered at two lattice constants to show the effect of compression. For the bct structures, the in-plane lattice constant was adapted to $\mathrm{MgO}$ and $c / a$ was relaxed. $T_{C}$ is calculated by a Monte Carlo method with exchange constants derived from the KKR or FLAPW methods.

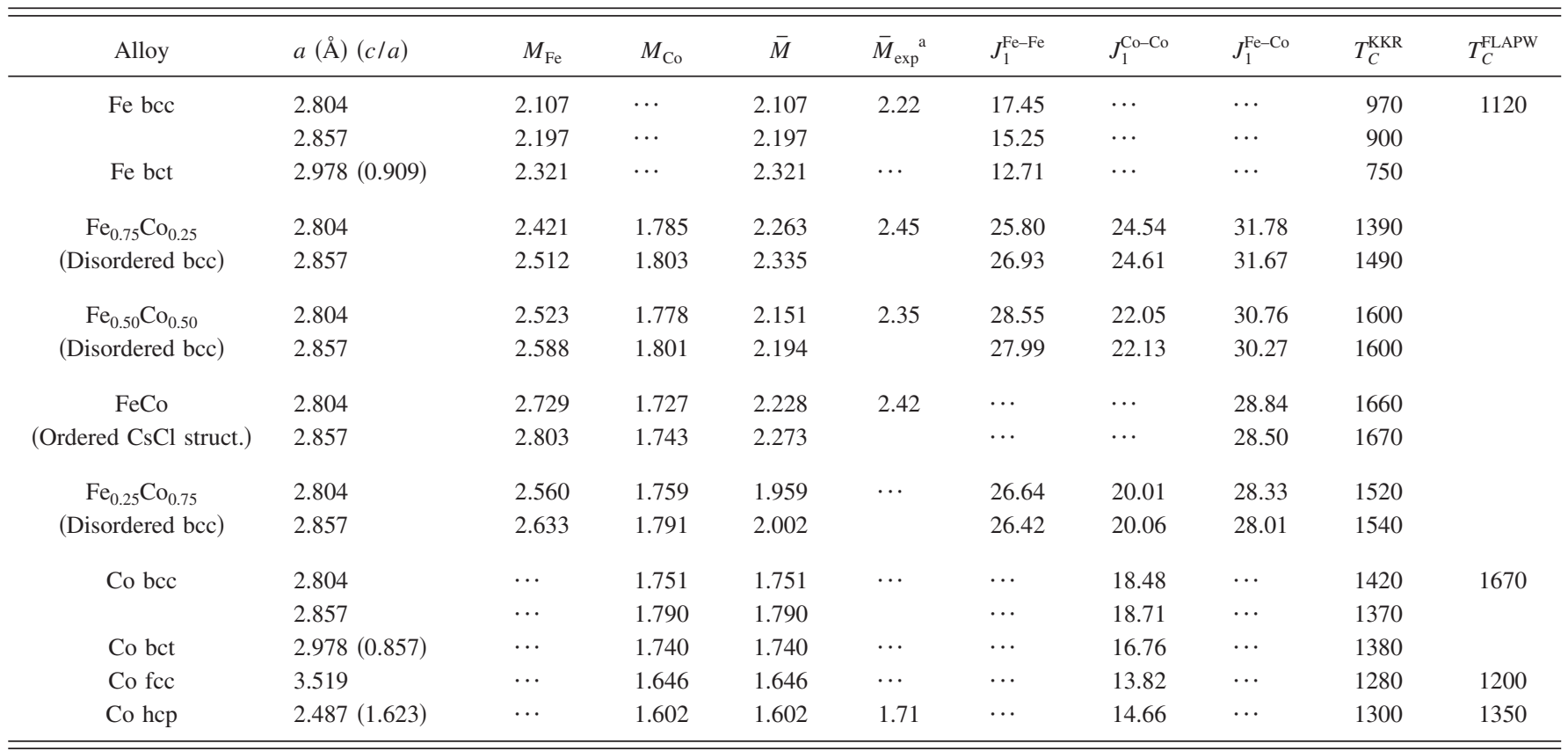

${ }^{\mathrm{a}}$ References 21 and 25 . 
tibility (and exchange constants $J_{i j}$ ) which is sensitive to $d^{\uparrow}-d^{\downarrow}$ Fermi-surface crossing or nesting ${ }^{29}$ because of virtual $d^{\uparrow}-d^{\downarrow}$ spin-flip excitations. In the case of strong ferromagnetism (Co or FeCo alloys), small structural perturbations cannot cause a $d^{\uparrow}-d^{\downarrow}$ charge transfer, because the $d^{\uparrow}$ band is fully occupied and well under $E_{F}$. Thus the exchange interactions are more stable in $\mathrm{Co}$ and $\mathrm{FeCo}$ alloys.

We proceed to the discussion of the exchange interactions and $T_{C}$ by introducing ${ }^{16}$ the coefficient $J_{0}=\Sigma_{i \neq 0} J_{0 i}$, corresponding to the band-energy cost for flipping the magnetic moment of a single atom, reflecting a "single-site spin stiffness." It is related to the mean-field Curie temperature via $k_{B} T_{C}^{\mathrm{MF}}=2 J_{0} / 3$ ( $k_{B}$ is Boltzmann's constant). (It is well known that mean-field theory overestimates $T_{C}$, but it is a useful tool for trends analysis.) By treating $E_{F}$ as a parameter, we calculate $T_{C}^{\mathrm{MF}}(E)$ as a function of band filling; in this way we are able to see the individual contribution of the states at each energy to the exchange interactions. In Fig. 1 we show $T_{C}^{\mathrm{MF}}(E)$, together with the DOS, for bcc $\mathrm{Fe}$ and Co. Evidently, $T_{C}^{\mathrm{MF}}(E)$ has a very similar form for $\mathrm{Fe}$ and Co, up to the approximately rigid band shift (it also has slightly higher values for $\mathrm{Fe}$ because of the stronger $d$ - $d$ hybridization); thus, $\mathrm{Fe}$ and $\mathrm{Co}$ can be compared in a unified picture. The negative values of $T_{C}^{\mathrm{MF}}(E)$, peaking around $-2 \mathrm{eV}$ for Co and $-1 \mathrm{eV}$ for $\mathrm{Fe}$, indicate an antiferromagnetic coupling, known from $\delta$-Mn. ${ }^{27}$ At higher energies the double-exchange mechanism sets in and $T_{C}^{\mathrm{MF}}(E)$ obtains strong positive contributions as $E$ crosses the final part of the $d^{\Uparrow}$ states and the antibonding $d^{\downarrow}$ states. Finally it drops to zero once the $d^{\downarrow}$ states are filled and the exchange mechanism is not present anymore.

In Fe, $E_{F}$ is located at a steep, ascending point of $T_{C}^{\mathrm{MF}}(E)$, before the maximum. Therefore, small structural perturbations resulting in band shifts have a strong influence on the $T_{C}$ of Fe, as seen in Table I. For Co, $T_{C}^{\mathrm{MF}}(E)$ is already descending at $E_{F}$ but is not as steep as for Fe. From this argument, Co is expected to have a higher and more robust $T_{C}$ than Fe. In an FeCo alloy $E_{F}$ is in-between, at the maximum of $T_{c}^{\mathrm{MF}}(E)$; then one expects the highest and most robust $T_{C}$, as is found by the Monte Carlo calculations.

In conclusion, ferromagnetism is found to be more robust in bcc Co than in bcc Fe. The $T_{C}$ of bcc Co is calculated to be the highest among all Co phases and stable with respect to structural changes even in the tetragonalized (bct) structures. This is advantageous in the temperature-dependent TMR of $\mathrm{Co} / \mathrm{MgO} / \mathrm{Co}$ MTJs compared to $\mathrm{Fe} / \mathrm{MgO} / \mathrm{Fe}$, as observed in recent experiments. ${ }^{11}$ Studies have shown ${ }^{30}$ that the TMR depends on the detailed interface properties, including the interface magnetization $M_{I}(T)$. Supported by the comparatively thick ferromagnetic back lead ( $>100 \mathrm{ML}$ ), the interface shows the same $T_{C}$ as the lead, which approaches quickly the bulk value. ${ }^{3}$

We suggest $\mathrm{FeCo} / \mathrm{bcc} \mathrm{Co} / \mathrm{MgO} / \mathrm{bcc} \mathrm{Co} / \mathrm{FeCo}(100)$ as an ideal junction for high TMR at room temperature. The reasons for this are as follows: (i) $\mathrm{FeCo}$ has the highest $T_{C}$ and is thus an ideal back lead. (ii) The $T_{C}$ of bcc Co is also very high (higher than $\mathrm{Fe}$ ), promising lower magnetic fluctuations at the interface. (iii) Epitaxial bcc- $\mathrm{Co} / \mathrm{MgO}(100)$ interfaces present excellent coherent spin-dependent transport properties, ${ }^{9,32}$ unlike a possibly disordered $\mathrm{FeCo} / \mathrm{MgO}$ interface. We therefore conclude that, at RT, the suggested junction has a higher TMR than $\mathrm{Fe} / \mathrm{MgO} / \mathrm{Fe}(100)$ or even $\mathrm{Fe} / \mathrm{bcc} \mathrm{Co} / \mathrm{MgO} / \mathrm{bcc} \mathrm{Co} / \mathrm{Fe}(100)$.

The authors gratefully acknowledge enlightening discussions with S. Yuasa and P. H. Dederichs.

${ }^{1}$ S. S. P. Parkin, C. Kaiser, A. Panchula, P. M. Rice, B. Hughes, M. Samant, and See-Hun Yang, Nat. Mater. 3, 862 (2004).

${ }^{2}$ S. Yuasa, T. Nagahama, A. Fukushima, Y. Suzuki, and K. Ando, Nat. Mater. 3, 868 (2004).

${ }^{3}$ K. Tsunekawa, D. D. Djayaprawira, M. Nagai, H. Maehara, S. Yamagata, N. Watanabe, S. Yuasa, Y. Suzuki, and K. Ando, Appl. Phys. Lett. 87, 072503 (2005); J. Hayakawa, S. Ikeda, F. Matsukura, H. Takahashi, and H. Ohno, Jpn. J. Appl. Phys., Part 2 44, L587 (2005).

${ }^{4}$ S. Ikeda, J. Hayakawa, Y. M. Lee, R. Sasaki, T. Meguro, F. Matsukura, and H. Ohno, Jpn. J. Appl. Phys., Part 2 44, L1442 (2005).

${ }^{5}$ E. Y. Tsymbal, O. N. Mryasov, and P. R. LeClair, J. Phys.: Condens. Matter 15, R109 (2003).

${ }^{6}$ T. Miyazaki and N. Tezuka, J. Magn. Magn. Mater. 139, L231 (1995); J. S. Moodera, L. R. Kinder, T. M. Wong, and R. Meservey, Phys. Rev. Lett. 74, 3273 (1995).

${ }^{7}$ S. Yuasa, T. Katayama, T. Nagahama, A. Fukushima, H. Kubota, Y. Suzuki, and K. Ando, Appl. Phys. Lett. 87, 222508 (2005).

${ }^{8}$ J. Mathon and A. Umerski, Phys. Rev. B 63, 220403(R) (2001); W. H. Butler, X. G. Zhang, T. C. Schulthess, and J. M. MacLaren, Phys. Rev. B 63, 054416 (2001).

${ }^{9}$ X. G. Zhang and W. H. Butler, Phys. Rev. B 70, 172407 (2005).

${ }^{10}$ L.-N. Tong, C.-L. Deng, F. Matthes, M. Müller, C. M. Schneider, and C.-G. Lee, Phys. Rev. B 73, 214401 (2006).

${ }^{11}$ S. Yuasa, A. Fukushima, H. Kubota, Y. Suzuki, and K. Ando, Appl. Phys. Lett. 89, 042505 (2006).

${ }^{12}$ M. Julliere, Phys. Lett. 54, 225 (1975).

${ }^{13} \mathrm{Ph}$. Mavropoulos, N. Papanikolaou, and P. H. Dederichs, Phys. Rev. Lett. 85, 1088 (2000); D. Wortmann, G. Bihlmayer, and S. Blügel, J. Phys.: Condens. Matter 16, S5819 (2004).

${ }^{14}$ H. Itoh, T. Ohsawa, and J. Inoue, Phys. Rev. Lett. 84, 2501 (2000); M. Ležaić, Ph. Mavropoulos, J. Enkovaara, G. Bihlmayer, and S. Blügel, ibid. 97, 026404 (2006).

${ }^{15}$ S. V. Halilov, H. Eschrig, A. Y. Perlov, and P. M. Oppeneer, Phys. Rev. B 58, 293 (1998).

${ }^{16}$ A. I. Liechtenstein, M. I. Katsnelson, V. P. Antropov, and V. A. Gubanov, J. Magn. Magn. Mater. 67, 65 (1987).

${ }^{17}$ J. P. Perdew and Y. Wang, Phys. Rev. B 45, 13244 (1992).

${ }^{18} \mathrm{H}$. Ebert and R. Zeller, SPR-TB-KKR package (http:// olymp.cup.unimuenchen.de/ak/ebert/SPR-TB-KKR).

${ }^{19}$ Implemented in the FLEUR code (http://www.flapw.de).

${ }^{20} T_{C}$ cannot be measured for bcc $\mathrm{Fe}_{1-x} \mathrm{Co}_{x}$ with high Co content, since these alloys transform to fcc at about $1200 \mathrm{~K}$ [R. Forrer, J. Phys. Radium 1, 49 (1930)]. hcp Co also transforms to fcc near 700 K (Ref. 21).

${ }^{21}$ R. M. Bozorth, Ferromagnetism (Van Nostrand, New York, 1951).

${ }^{22}$ W. C. Ellis and E. S. Greiner, Trans. Am. Soc. Met. 29, 415 (1941); G. A. Prinz, Phys. Rev. Lett. 54, 1051 (1985).

${ }^{23}$ D. Bagayoko, A. Ziegler, and J. Callaway, Phys. Rev. B 27, 7046 (1983).

${ }^{24}$ Calculations show that the moment of bcc Co is also stable against noncollinear magnetic distortions [L. M. Sandratskii and J. Kübler, Phys. Rev. B 47, 5854 (1993)].

${ }^{25}$ D. I. Bardos, J. Appl. Phys. 40, 1371 (1969).

${ }^{26}$ M. F. Collins and J. B. Forsyth, Philos. Mag. 8, 401 (1963).

${ }^{27} \mathrm{~J}$. Kübler, Theory of Itinerant Electron Magnetism (Oxford University Press, New York, 2000).

${ }^{28}$ A. Díaz-Ortiz, R. Drautz, M. Fähnle, H. Dosch, and H. M. Sanchez, Phys. Rev. B 73, 224208 (2006); A. Y. Liu and D. J. Singh, ibid. 46, 11145 (1992); K. Schwarz and D. R. Salahub, ibid. 25, 3427 (1982).

${ }^{29}$ R. Lizárraga, L. Nordström, L. Bergqvist, A. Bergman, E. Sjöstedt, P. Mohn, and O. Eriksson, Phys. Rev. Lett. 93, 107205 (2004).

${ }^{30}$ E. Y. Tsymbal and K. D. Belashchenko, J. Appl. Phys. 97, 10 C910 (2005); J. S. Moodera, J. Nowak, and R. J. M. van de Veerdonk, Phys. Rev. Lett. 80, 2941 (1998)

${ }^{31}$ F. Huang, G. J. Mankey, M. T. Kief, and R. F. Willis, J. Appl. Phys. 73, 6760 (1993); R. Schiller and W. Nolting, Solid State Commun. 110, 121 (1999).

${ }^{32}$ C. Heiliger, M. Gradhand, P. Zahn, and I. Mertig (unpublished). 